\title{
Non-symmetrical line broadening effects using short-pulse QCL spectrometers as determined with sub-nanosecond time-resolution
}

\author{
S. Welzel · J. Röpcke
}

Received: 30 December 2009 / Revised version: 7 May 2010 / Published online: 19 June 2010

(C) The Author(s) 2010. This article is published with open access at Springerlink.com

\begin{abstract}
Quantum cascade lasers (QCLs) have attracted considerable interest as an alternative tuneable narrow bandwidth light source in the mid-infrared spectral range for chemical sensing. Pulsed QCL spectrometers are often used with short laser pulses and a bias current ramp similar to diode laser spectroscopy. Artefacts in the recorded spectra such as disturbed line shapes or underestimated absorption coefficients have been reported. A detailed time-resolved high-bandwidth analysis of individual pulses during a laser sweep has been performed. Quantitative results for $\mathrm{CH}_{4}$ absorption features around $1347 \mathrm{~cm}^{-1}(7.42 \mu \mathrm{m})$ fell short of the expected values for reasonable operating conditions of the QCL. The origin of the artefacts using short pulses was identified to be partly of the same nature as in the case of long laser pulses. A complex combination with the tuning principle was found, leading to an apparently increased instrumental broadening (effective line width) and underestimated concentrations at low-pressure conditions.
\end{abstract}

\section{Introduction}

Chemical sensors based on the absorption of light are a powerful diagnostic tool for quantifying gaseous species concentrations. The mid-infrared (MIR) spectral range (3-20 $\mu \mathrm{m})$

S. Welzel $(\bowtie) \cdot$ J. Röpcke

INP Greifswald, Felix-Hausdorff-Str. 2, 17489 Greifswald, Germany

e-mail: welzel@physik.uni-greifswald.de

Fax: +31-40-2456442

Present address:

S. Welzel

Eindhoven University of Technology, P.O. Box 513, 5600 MB

Eindhoven, The Netherlands with strong ro-vibrational absorption features is therefore preferably used for trace gas sensing, plasma diagnostics in research and industrial environments or medical diagnostics. Semiconductor laser sources which can provide the required continuous tuneability and low instrumental broadening to achieve a high selectivity have been limited to tuneable diode lasers (TDLs) based on lead salts for years. In particular, the advent of quantum cascade lasers (QCLs) $[1,2]$ and their commercial availability have increased the number and variety of MIR spectroscopic laser applications. While the first laser devices could be used at room temperature only in pulsed mode at low duty cycles, room temperature continuous wave (cw) lasers have recently been available due to an improved thermal management. QCLs are now considered as a valuable alternative light source for the MIR spectral region and as a substitute to TDLs [3].

Thus, early experiments with pulsed QCLs straightforwardly combined short laser pulses with the conventional method for sweeping lead salt lasers in TDL absorption spectroscopy (TDLAS) by ramping a DC current [4-6]. This short-pulse mode of operation is often referred to as inter pulse method. Extensions originally developed for TDLAS, e.g., the sweep integration method [7, 8], were adapted to pulsed QCL spectrometers [9] and enable a time-resolution of milliseconds. Employing this method is not only of interest for on-line trace gas measurements, on which most of the experiments carried out so far were focussing [5, 6 , 9-17], but also for monitoring species in plasma diagnostics [18-20]. Later, the inherent frequency-down chirp of the lasers was exploited by using long pulses to acquire absorption spectra during the pulse [21,22].

Although the successful implementation of QCL absorption spectroscopy (QCLAS) was very often reported, obstacles in the spectra along with pulsed QCLs have been recognised. Apart from pulse-to-pulse fluctuations inherent to the 
pulsed operation, spectral line broadening was identified as a main challenge while using the inter pulse mode for highly sensitive detection schemes [10]. Effective laser line widths (full width at half maximum-FWHM) between 0.0095 $\mathrm{cm}^{-1}(290 \mathrm{MHz})$ and $0.093 \mathrm{~cm}^{-1}(2.79 \mathrm{GHz})$ have been reported. According to McCulloch et al. the spectral resolution of QCL spectrometers is determined both by the chirp rate and the pulse width of the lasers [23]. Particularly, shortpulse spectrometers may approach the Fourier limit (constant bandwidth-time product) for extremely short pulses (5 ns, $290 \mathrm{MHz}$ [5]). On the other hand, increased pulse widths lead to a chirp-limited resolution $(70 \mathrm{~ns}, 2.79 \mathrm{GHz}$ [6]). Furthermore, asymmetric line shapes of absorption features have been detected in several studies employing both the short-pulse [9, 11, 24] and the long-pulse mode [25]. Additionally, the latter method suffers from non-linear absorption effects [26]. The majority of these issues are not known from conventional TDLAS and degrade the performance of QCL spectrometers. This is particularly of importance at lower pressures, since under such conditions the laser line width of pulsed QCLs was found to exceed the typical Doppler broadening [27] due to the frequency chirp. Non-linear effects (e.g., fast passage) appeared also more pronounced [26].

A few studies have been concerned with these drawbacks, however, a consistent explanation is absent so far, although all obstacles seem to be connected with the chirped laser. While a theoretical description for the non-linear absorption phenomena based on optical Bloch equations exists [25, $26,28]$, the origin of distorted line shapes in the short-pulse mode is not yet completely understood [9]. The problem is often empirically minimised by finding a compromise between a reasonable signal-to-noise ratio (SNR) and a narrow spectral width [12]. The sensitivity of spectrometers or quantitative results are typically verified by calibrating an effective laser line width. Another approach abstracts correction factors by comparing measurements and simulated spectra [e.g., 6, 9, 13]. Only in a few studies the results were validated with independent counter-measurements by means of TDLs [9, 11] or cw QCLs [12, 29]. Recently, an intercomparison between the short- and long-pulse method has been in the centre of interest [14]. Unfortunately, this study was limited to pressure ranges (hundreds of mbar) where the artefacts diminish or disappear. Finally, the asymmetrical line shapes have recently been recognised as an effect of the detector bandwidth which smoothes out particularly the rapid passage features [24].

A more detailed analysis of particularly laser line width effects in the inter pulse mode was carried out by Nelson et al. [9]. However, extracting accurate laser line widths from their experimental spectra was difficult, because a strong asymmetry of absorption lines was found. This effect was amplified by applying higher operating voltages, although the simultaneously recorded electrical pulse shapes showed no significant difference. Consequently, Nelson et al. concluded that a QCL should be operated close to its threshold with short pulses close to the uncertainty broadening limit with rise times faster than $8 \mathrm{~ns}$ achieved with the original Alpes Lasers operation unit. The investigation of asymmetrically broadened absorption lines was continued by McManus et al. since such absorption features revealed another phenomenon: the peak absorption signal decreased as the distortion became more pronounced [12]. In these experiments the operating voltage was varied at constant pulse width. McManus et al. suggested the product of peak absorbance and detector signal as a figure of merit for determining the optimum operating conditions. Since the latter factor increases with higher QCL voltages this product levels off which was chosen as the operation point.

Nevertheless, several questions remained unclear from these studies. Firstly, although Nelson et al. worked at low pressure (4 Torr) no rapid passage signatures were found. Secondly, the asymmetric absorption lines in the inter pulse spectra exhibited both a high-frequency tail $[9,11]$ and a low-frequency tail [12]. If just a single frequency-down chirped QCL pulse is considered, the rapid passage structures are always observed after the absorption feature, i.e. at the lower frequencies. On the other hand, in the inter pulse mode of operation the current is usually ramped in such a way that the QCL frequency increases with time (cf. Sect. 2.1). A straightforward convolution of a molecular absorption line which is scanned from lower to higher frequencies with rapid passage oscillations which appear later in time would lead to the conclusion that a high-frequency tail can be expected. A potential pulse-to-pulse frequency jitter or not yet identified properties of the individual pulse were proposed [9].

The present study employing the inter pulse method under low-pressure conditions focussed on a detailed analysis of each short chirped pulse. Section 2 describes the experimental approach encompassing the time-resolved detection of individual chirped pulses during a laser sweep. The analysis of the results (Sect. 3) reveals a complex combination of the sweep approach in the short-pulse mode with those obstacles known from the long-pulse method. This may also serve as an explanation for the lower-frequency shape of absorption lines and is summarised in Sect. 4.

\section{Experimental}

\subsection{Experimental arrangement}

All experiments were carried out in a two-laser configuration (Fig. 1) encompassing two co-aligned TDLAS and QCLAS arrangements. Details of the tuneable diode laser 


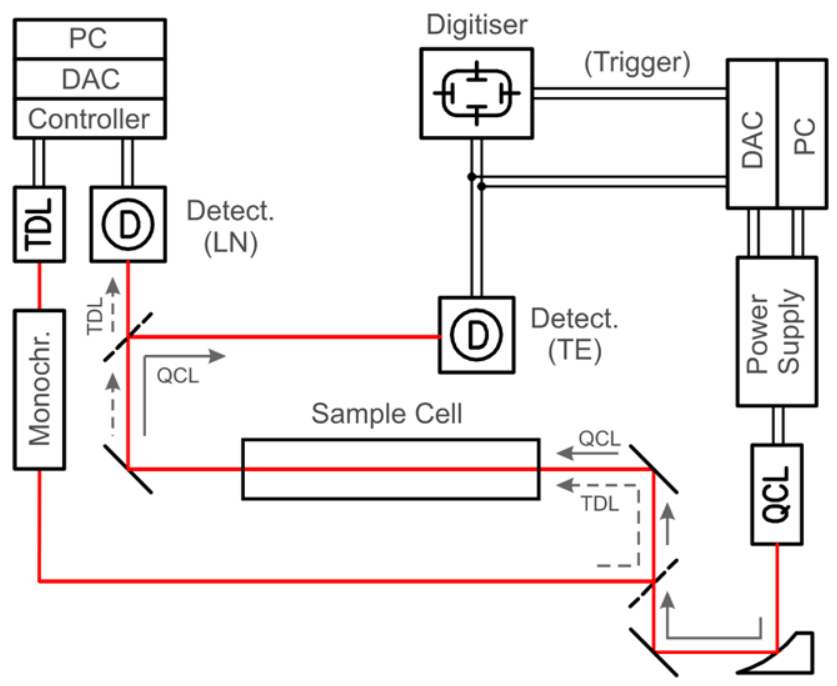

Fig. 1 Two laser arrangement consisting of co-aligned TDL and QCL spectrometers. Flip mirrors (dashed) are used to switch between the systems. Double lines represent electric connexions, single lines the optical beam path which is additionally indicated by arrows. (DAC-data acquisition card, TE/LN Detect.- thermoelectrically/liquid nitrogen cooled detector)

Table $1 \mathrm{CH}_{4}$ line positions covered by the two laser arrangement and respective line strengths [33]

\begin{tabular}{clc}
\hline$\#$ & $\begin{array}{l}\text { Line position } \\
{\left[\mathrm{cm}^{-1}\right]}\end{array}$ & $\begin{array}{l}\text { Line strength } \\
{[\mathrm{cm} / \text { molecule }]}\end{array}$ \\
\hline & 1346.5755 & $2.306 \times 10^{-20}$ \\
& 1346.7396 & $3.460 \times 10^{-20}$ \\
& 1347.0543 & $5.775 \times 10^{-20}$ \\
I & 1347.8016 & $3.381 \times 10^{-22}$ \\
II & 1347.9214 & $3.466 \times 10^{-20}$ \\
III & 1348.0416 & $3.470 \times 10^{-20}$ \\
IV & 1348.1525 & $1.642 \times 10^{-22}$ \\
\hline
\end{tabular}

InfraRed Multi-component Acquisition system (IRMA) can be found elsewhere [30]. Since the lead salt laser could not be tuned to cover exactly the same $\mathrm{CH}_{4}$ lines as the QCL similar strong $\mathrm{CH}_{4}$ features around $1346.5 \mathrm{~cm}^{-1}$ were chosen for quantitative comparisons and are summarised in Table 1 . Strictly speaking, the present study concerned pure $\mathrm{CH}_{4}$ samples which did not necessarily have to be confirmed by TDLAS. However, in follow-up experiments, which will be considered in another publication [31,32], $\mathrm{CH}_{4}$ admixtures in a background gas were used and thus quantified by the IRMA system.

The distributed feedback QCL (Alpes Lasers) was operated in a temperature stabilised housing and driven with a commercial QCL measurement and control system (QMACS, neoplas control). The laser was triggered using a computer controlled data acquisition card (DAC). The fre-

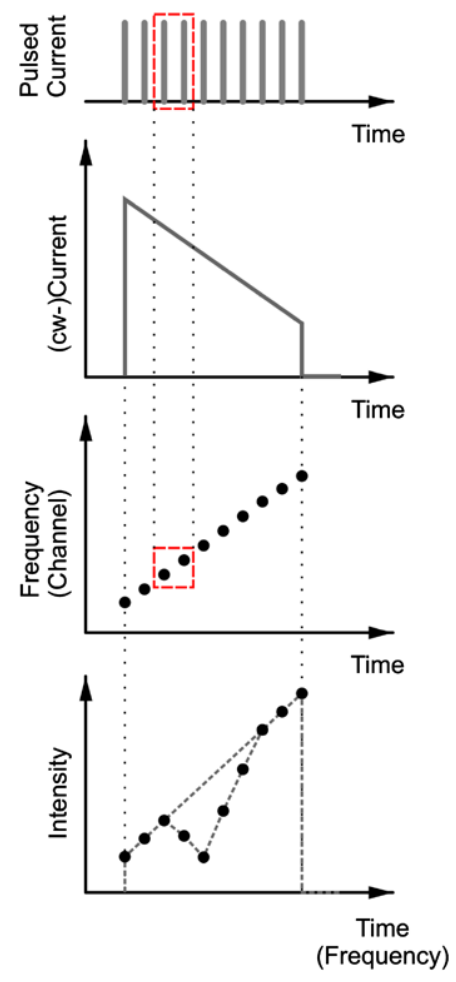

Fig. 2 Schematic diagram of the inter pulse mode: Spectral tuning is accomplished by impressing a sub-threshold current ramp with a negative gradient on the pulsed QCL. The negative temperature tuning coefficient of the laser yields an increase in its emission frequency. The laser intensity decreases simultaneously. Each QCL pulse corresponds to a data point in an absorption spectrum (lower panel). Two laser pulses are marked with a dashed box and are enlarged in Fig. 3

quency scan of the QCL in the short-pulse mode is achieved by a current ramp (Fig. 2) which was generated from an externally supplied voltage ramp provided by the software package TDL Wintel in combination with the DAC. The program sweeps the laser, acquires the absorption spectra and analyses them simultaneously [9]. In order to achieve an increase in the laser frequency with time the sub-threshold tuning current is commonly decreased during the ramp (Fig. 2). This follows from the negative temperature tuning coefficient of QCLs. Simultaneously the emitted laser intensity increases with time (Fig. 2). A short gate-off period at the end of each sweep suppresses the laser emission for measuring the detector offset voltage. The details of the presently used data acquisition and laser synchronisation principle for the inter pulse mode are essentially the same as described by Nelson et al. [10] except for the QCL driver (Q-MACS instead of the original Alpes Lasers Starter Kit).

For the experiments presented here a single sweep consisted of 230 and 20 laser pulses during the gate onand off-period respectively. The employed QCL repetition frequency of $100 \mathrm{kHz}$ yielded therefore a sweep rate of $0.4 \mathrm{kHz}$. The QCL emission range was tuned to $1347.75-$ 


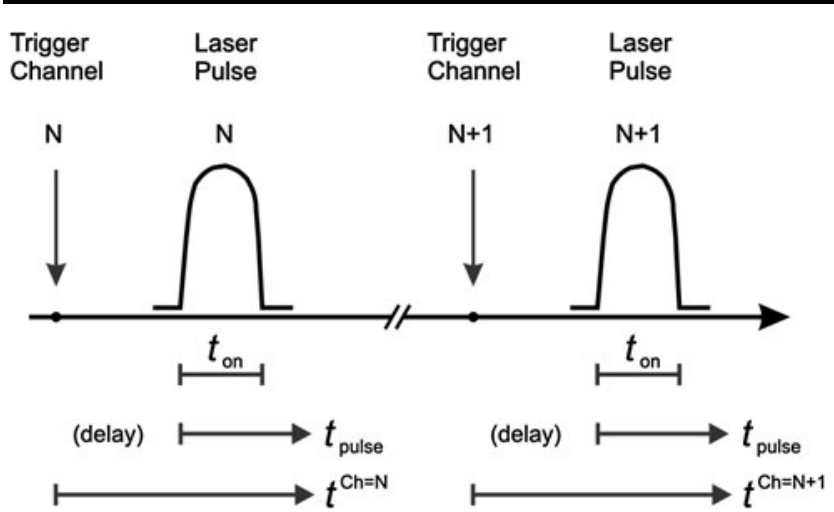

Fig. 3 Schematic example of two subsequent QCL pulses (i.e. channels $N$ and $N+1$ ) of length $t_{\text {on }}$ during a laser sweep using the inter pulse mode. Two different time scales, i.e. a time scale $t^{\mathrm{Ch}}$ for each channel and an intrinsic time $t_{\text {pulse }}$ for the laser pulse, are introduced for further data analysis

$1348.25 \mathrm{~cm}^{-1}$ covering $4 \mathrm{CH}_{4}$ absorption features which are labelled as I-IV in what follows (Table 1).

The QCL pulses were detected with a thermoelectrically (TE) cooled detector encompassing a high-bandwidth preamplifier (neoplas control, VIGO element PDI-2TE$10.6,0.25 \times 0.25 \mathrm{~mm}^{2}$ ). In this case the detection bandwidth was limited by the preamplifier to about $250 \mathrm{MHz}$. It is known that TE cooled detectors may exhibit a nonlinear response function. It has been confirmed in preliminary experiments-though scrutinising such effects was beyond the scope of this study - that non-linear behaviour is negligible for the present combination of detector element and preamplifier as long as the detector signal is lower than $\sim 75 \%$ of the maximum detector voltage. Special attention has been paid to fulfill this criterion. For the timeresolved single-pulse analysis the data acquisition by the DAC was replaced by a fast digitising oscilloscope (LeCroy WR104Xi).

The radiation from either the TDLAS or QCLAS arrangement and its corresponding detector could be chosen by means of retractable mirrors (Fig. 1). The laser beam was directed to a reference cell of $0.15 \mathrm{~m}$ length filled with $10 \mathrm{mbar}$ $\mathrm{CH}_{4}$. This pressure was still sufficiently low to observe rapid passage structures in the (long-) pulse spectra of the pulsed QCL.

\subsection{Time-resolved detection}

In the conventional implementation of the inter pulse method each QCL pulse represents a spectral data point (or channel) as detailed in Fig. 2. The DAC thereby samples a value of the detector output being usually averaged over the entire pulse duration. For the time-resolved analysis all 230 pulses were sampled with a (high) time-resolution of $0.1 \mathrm{~ns}$ during the gate-on phase.

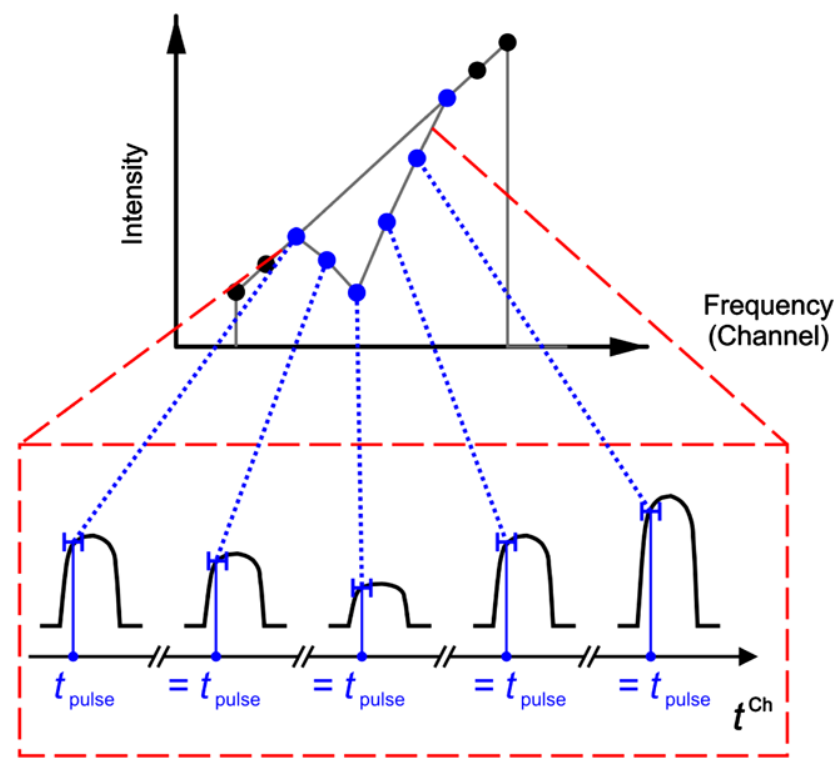

Fig. 4 Data analysis procedure carried out for the high time-resolution experiments. Each QCL pulse (channel) is sampled at the same $t_{\text {pulse }}$ (lower panel). The corresponding detector signal represents a spectral data point as indicated for a few points in the upper panel

The post-measurement analysis of each laser pulse required a thorough treatment of the different time scales during a laser sweep. Each laser pulse (or channel) was therefore described by its own time scale $t^{\mathrm{Ch}}$ starting at zero after the corresponding trigger event. This is illustrated in Fig. 3 for two subsequent channels $N$ and $N+1$. The start and the end of all individual QCL pulses were determined on this time scale $t^{\mathrm{Ch}}$. A detector signal just above noise level was chosen as criterion for determining both values. The difference yielded the effective (optical) pulse width $t_{\text {on }}$ (Fig. 3) which could be compared with the nominal current pulse width. Finally, another time scale, $t_{\text {pulse }}$, was introduced to eliminate the delay and jitter between trigger and laser pulse mainly caused by the electronics and the optical beam path (Fig. 3). In this way all individual pulses could be compared and further analysed on the basis of a common time scale $t_{\text {pulse }}$ which was set to zero when the QCL pulse starts. Table 2 summarises the established definitions for the different time scales. It should be mentioned that both $t^{\mathrm{Ch}}$ and $t_{\text {pulse }}$ serve as time scales whereas $t_{\mathrm{on}}$ represents the value of the experimentally observed pulse width.

The post-measurement analysis is illustrated in Fig. 4 for the schematic absorption feature shown in the lower panel of Fig. 2. Each acquired spectral data point (i.e. laser pulse) was scrutinised (for experimentally observed laser pulses see Fig. 9). Firstly, the beginning of the QCL pulse and thus the time scale $t_{\text {pulse }}$ was established. Secondly, the detector signal at $t_{\text {pulse }}=$ const. was determined. For this purpose 3 adjacent points were averaged (Fig. 4) resulting in an effective time-resolution of $0.3 \mathrm{~ns}$. Plotting these detector signals 
Table 2 Time scale definitions employed for the analysis of individual QCL pulses

\begin{tabular}{ll}
\hline Symbol & Definition \\
\hline$t^{\mathrm{Ch}}$ & Time scale of each pulse \\
& $t^{\mathrm{Ch}}=0$ at the trigger event \\
$t_{\text {pulse }}$ & Time scale of each pulse without delays inherent to the system \\
& $t_{\text {pulse }}=0$ at begin of the laser pulse \\
$t_{\mathrm{on}}$ & Experimentally determined optical pulse width \\
& $t_{\mathrm{on}}=t^{\mathrm{Ch}}$ (pulse end $)-t^{\mathrm{Ch}}$ (pulse begin) \\
\hline
\end{tabular}

as a function of the channel number yields several absorption spectra at $t_{\text {pulse }}=$ const. (cf. upper panel of Fig. 4 and Fig. 7).

\section{Results and discussion}

\subsection{Conventional approach}

At first, the characteristics of the tuning method in combination with the standard configuration (i.e. detector signal measured with DAC and displayed with TDL Wintel) was established. In contrast to Nelson and McManus et al. the laser was operated with a different driver unit (Q-MACS) [9, 12]. The pulsed current through the laser was thereby not directly accessible. For that purpose complementary measurements were performed with a commercial miniature wideband current probe (IST). The current was monitored as close as possible to the laser using the internal wiring in the QCL housing. Similar to earlier measurements, a rise time of 8 ns was confirmed with this approach for an Alpes Lasers Starter Kit [9]. On the other hand the presently used pulse unit yielded a faster increase of the current pulse of $\sim 2$ ns.
Nelson's idea to reduce the laser chirp with shorter pulses [9] is thus realised.

QCL transmission spectra of the reference cell are shown in Fig. 5 for different operating voltages up to $0.6 \mathrm{~V}$ above threshold $(11.5 \mathrm{~V})$ and a constant pulse width of $20 \mathrm{~ns}$. The central heat sink temperature was kept constant throughout all measurements in order to reduce thermally induced changes of the QCL pulses. On the other hand, the spectral sweep close to the threshold voltage was then limited and could not cover all $\mathrm{CH}_{4}$ features (I-IV). Instead of the expected typical oscillatory structure of the rapid passage effect another distortion of the absorption lines is found in the spectra. A strong asymmetry at the low-frequency side of the line, particularly at voltages clearly above threshold, is observed and hampers a reasonable fit to line profiles for a quantitative analysis. The absorption lines are broadened by features similar to a shadow of the original line. This is clearly visible at $0.4 \mathrm{~V}$ above threshold for line III, i.e. at the end of the current ramp, and at slightly higher operating voltages for line II. These additional absorption-like features at higher QCL voltages cannot be explained by molecular transitions which would also be independent from the QCL operating conditions. Additionally, the peak absorption de-

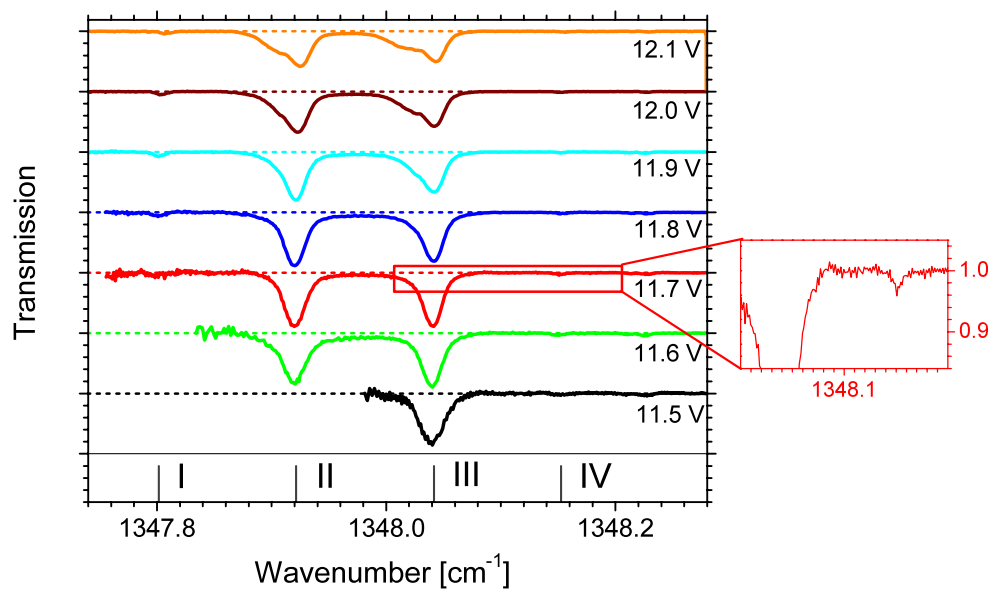

Fig. 5 Conventionally acquired inter pulse mode spectra of $10 \mathrm{mbar}$ $\mathrm{CH}_{4}(0.15 \mathrm{~m}$ absorption path). The measurements were performed at constant pulse width $(20 \mathrm{~ns})$ of the QCL current, constant heat sink temperature and variable QCL voltages as indicated. Transmission spectra are stacked for clarity and the corresponding zero signal level is indicated (dashed line). Absorption features which are used for further analysis are marked with I-IV (Table 1). The inset illustrates the achievable SNR for weaker lines (e.g., IV) 


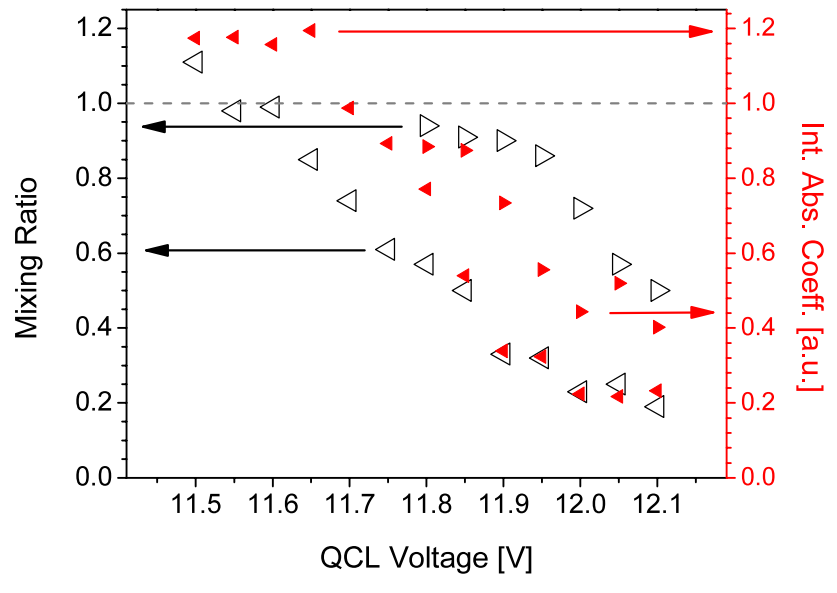

Fig. $6 \mathrm{CH}_{4}$ mixing ratios deduced for the absorption features I (triangles left) and IV (triangles right) for all applied QCL voltages between

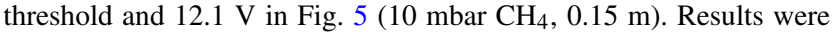
obtained from a fit to a Voigt profile (open symbols, left hand scale) and from the integrated absorption coefficient (full symbols, right hand scale). The nominal mixing ratio $(=1)$ was confirmed by TDLAS (dotted)

creases as the distortion becomes more pronounced. Only at operating voltages close to the threshold of the QCL (Fig. 5) the absorption line appears symmetrically, but at the expense of a considerably reduced detector signal. This in turn leads to a poor SNR and the weak features I and IV vanish below their detection threshold. Similar obstacles have already been reported $[9,12]$. Since another QCL driver was used here it can be assumed that the observed effects are not directly connected to the current pulses (shape, rise time, etc.).

The acquired spectra were quantitatively analysed by applying two independent methods. Firstly, TDL Wintel inferred the $\mathrm{CH}_{4}$ mixing ratio from a least-square fit of the absorption lines to Voigt profiles calculated by using pressure, temperature, effective laser line width and line strengths as input parameters. Secondly, the mixing ratios were determined from the integrated absorption coefficient, i.e. integrating the entire absorption line. In the latter case no line profile or effective laser line width has to be assumed and the distorted features are entirely captured. However, it could be argued that the latter method may not facilitate the calculation of correct mixing ratios if a spectrally broadened laser source is used. For this reason, only the integrated absorption coefficient is used and compared with the results from TDL Wintel. Both results are displayed for $\mathrm{CH}_{4}$ lines I (if detectable in the sweep) and IV in Fig. 6. No significant difference between the relative behaviour of both analysis approaches is observed. Although the same sample was studied the $\mathrm{CH}_{4}$ mixing ratio or the integrated absorption coefficient drops as the QCL voltage is increased. The expected concentration of $100 \%$ is only obtained for the weak feature IV at QCL voltages close to the threshold. If the operating voltage is increased the deduced mixing ratios decrease to $\sim 50 \%$ for line I and even down to $20 \%$ for line IV which appears later throughout the sweep.

The difference in the relative behaviour between both analysis approaches may be especially due to an independent determination of the baseline, but does not significantly change the conclusion. A straightforward zero absorbance measurement to accurately determine the baseline was not feasible with the sample cell used here, but it has been considered in the follow-up experiments [31]. Therefore the fit in TDL Wintel was tuned in such a way that the baseline slightly overestimates the mixing ratio. Hence, the quantitative results in Fig. 6 should be considered as an upper limit. At higher QCL voltages the integration method might generally be better suited to account for the distortion. Note that the fit results are not considerably improved if the effective laser line width is adjusted in a range which yields a reasonable fit to the acquired spectra (i.e., $0.012-0.020 \mathrm{~cm}^{-1}$, FWHM). In all cases a (true) mixing ratio of 1 was confirmed by the TDL system.

Considering the strong absorption features II and III a quantitative analysis is hampered due to their strong absorption (optically thick lines). However they are perfectly suited to illustrate qualitatively the effects of (asymmetrical) line broadening or reduced peak heights. Since QCLs exhibit increased (peak) power values compared to TDLs non-linear power saturation effects may play a role for strong features. However, it is clear from this quantitative analysis that even for weak absorption features, where potential power saturation can be neglected in a first approximation, the (upper limit) integrated absorption coefficient and hence the concentration values are underestimated for relevant QCL operating conditions and output power values. The picture does not change if the current pulse width is reduced from $20 \mathrm{~ns}$ down to $12 \mathrm{~ns}$ (minimum) while keeping the QCL voltage near the simultaneously changing threshold. This also suggests that a chirp-limited spectral resolution might be only a part of the explanation, particularly for the asymmetrical line broadening. Since the frequency chirp and thus a certain effective spectral broadening is inherent to pulsed QCLs, the strong influence of the QCL operating conditions has to be further scrutinised.

\subsection{Spectral deconvolution of individual pulses}

All previous experiments and their analysis suggested that a deeper insight into the intrinsic properties of the laser pulses is essential to describe the observed effects. For this purpose the detector signals of the individual QCL pulses were analysed at a constant time $t_{\text {pulse }}$ and plotted against their channel number (Fig. 7). Different $t_{\text {pulse }}$ covering the full laser pulse width were analysed, i.e. Fig. 7 represents only one laser sweep which was deconvolved into several $t_{\text {pulse }}$. Additionally, the detector signal was averaged over the entire pulse width (Fig. 7, upper panel). All measurements 


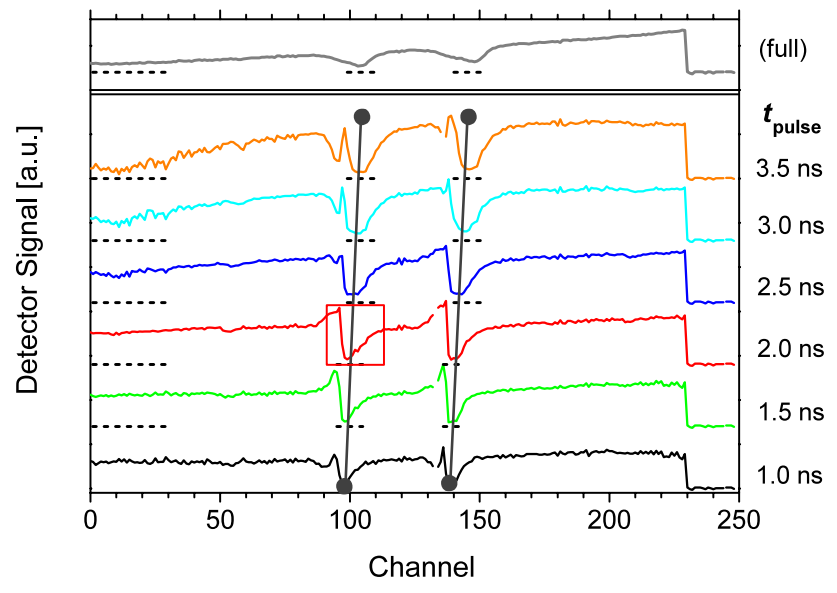

Fig. $7 \mathrm{CH}_{4}$ absorption spectra (10 mbar, $\left.0.15 \mathrm{~m}\right)$ retrieved from the individual detector signals of the chirped QCL pulses during a laser sweep. Spectra are deduced from the same sweep. Upper panel: Averaged signal over the entire pulse. Lower panel: Detector signals are sampled at different $t_{\text {pulse }}=$ const. (right hand scale) and stacked for clarity. The zero level from the gate-off period is indicated by dotted lines. The two solid lines represent the shift of spectral features with increasing $t_{\text {pulse }}$. Individual QCL pulses within the marked box are enlarged in Fig. 9

were carried out with the same $\mathrm{CH}_{4}$ sample cell and under similar operating conditions as for the conventional approach in Fig. 5 using a moderate QCL voltage about $0.2 \mathrm{~V}$ above threshold (20 ns QCL current pulses). In this way a reasonable output power of the laser could be achieved and first line shape distortions were observed. In what follows the discussion and conclusions are based on these experimental conditions as an example though they can be generalised, since a complementary measurement series at the threshold does not change the picture [32].

The compilation of deconvolved spectra (Fig. 7) reveals several key features, (i)-(iv), of the obstacles and hence of the short-pulse tuning method:

(i) The rapid passage effect is also present in low-pressure spectra.

(ii) The time-averaged signal exhibits the same distorted line shapes without rapid passage oscillations as observed with the conventional approach (Fig. 5).

(iii) The peak absorption decreases at higher $t_{\text {pulse }}$ and in the averaged signal.

(iv) The position of spectral features (i.e. channel number) shifts with $t_{\text {pulse }}$.

The typical rapid passage structure at the low-frequency side of a line, which is expected under low-pressure conditions with pulsed QCLs, is not seen in the spectra acquired with the conventional DAC equipment. However it is present in the deconvolved spectra (i) and the amplitude of the oscillations decreases as $t_{\text {pulse }}$ approaches the end of the short pulse, because the chirp rate typically drops considerably during the first nanoseconds of a pulse. The variable chirp rate is accompanied by an increase of the output power of the QCL at higher $t_{\text {pulse }}$ which may cause additional power saturation, at least for the strong features II and III. Consequently the peak absorption decreases in those spectra retrieved from the end of the short-pulse (iii). The shift of spectral features (iv) is linked with the chirp of the pulse and the sign of the current ramp slope. After the sign of the slope was reversed, i.e. decreasing laser frequency and intensity during the sweep, the shadow was observed at higher channels (see also [9, 11]). A detailed analysis of the shift of a selected spectral position reveals an uncertainty of at least $8-10$ channels. This corresponds to a shift of $\sim 0.025 \mathrm{~cm}^{-1}$ $(750 \mathrm{MHz})$ for the present experimental conditions and accords well with the effective line widths found in earlier studies.

Furthermore, it is obvious from Fig. 7 that contributions to the spectrum from high $t_{\text {pulse }}$ values are overestimated in the time-averaged detector signal (Fig. 7, upper panel) due the higher output power at this time. Consequently, the averaged absorption profile appears asymmetrically broadened with a shadow of the line at lower channels. In an idealised picture a convolution of an absorption feature with a rapid passage structure-appearing at the right hand side of a line-could explain a high-frequency tail in the inter pulse mode. Figure 7 therefore reveals that this straightforward assumption must fall short of explaining the asymmetry in recorded spectra. Since the rapid passage oscillations are always present at the low-frequency side of a line the sign of the current ramp slope and the laser intensities of the individual pulses determine the appearance of the shadow. However, the chirp rate of the QCL can also affect this artefact, which will be discussed below.

It is clear that this artificial asymmetric instrumental broadening is difficult to describe analytically and reduces the peak absorption in addition to potential power saturation effects. Thus, concentration values are further underestimated and weak absorption features are dropped below the noise level (e.g. features I and IV in Fig. 7 (upper panel) and Fig. 5). Averaging also eliminates rapid passage structures in the spectra (ii) and considerably degrades the quality of etalon spectra [32].

The analysis of the start and end point of the emitted pulse (Fig. 8) yielded a considerable scatter in the $t^{\mathrm{Ch}}$ data of $\pm 2 \mathrm{~ns}$ whereas the corresponding difference (optical pulse width $t_{\text {on }}$ ) showed only a small uncertainty of $\pm 0.6 \mathrm{~ns}$. This indicates that mainly jitter between the trigger event and the laser emission causes the strong fluctuations in $t^{\mathrm{Ch}}$ (which motivated the introduction of a second time scale $t_{\text {pulse }}$ (Table 2)). Figure 8 illustrates that the optical pulses appear earlier and fade away later throughout the sweep. Consequently the pulse width rises from $4 \mathrm{~ns}$ to $10 \mathrm{~ns}$ during the laser sweep and is, hence, clearly smaller than the nominal 


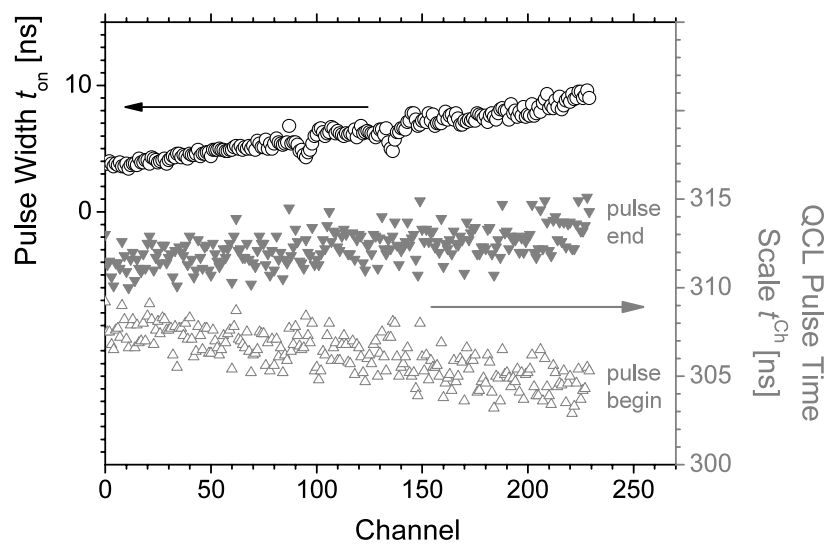

Fig. 8 Start (triangle up) and end (triangle down) point of all QCL pulses in Fig. 7 based on the time scale $t^{\mathrm{Ch}}$ (right hand scale, grey) and the corresponding difference yielding the pulse width $t_{\text {on }}$ (circle, left hand scale, black)

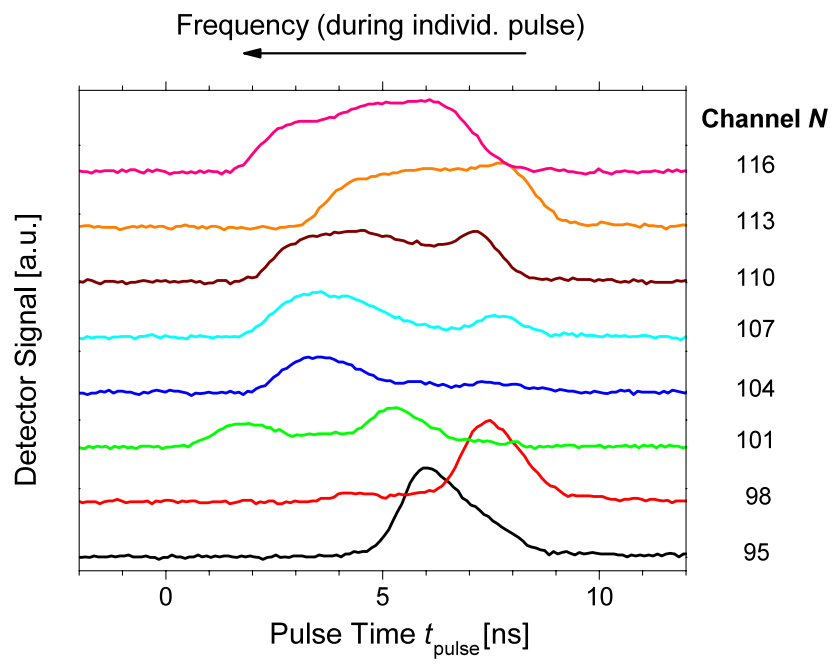

Fig. 9 Time-resolved spectra of single frequency-down chirped pulses during the inter pulse laser sweep in Fig. 7 (highlighted with a box). The corresponding channel numbers $N$ for the selected examples around the $\mathrm{CH}_{4}$ absorption feature II are indicated on the right hand scale

current pulse width. This behaviour emanates from the temperature tuning via the DC current ramp while the QCL operating voltage, i.e. the pulsed QCL current, is kept constant. The temperature is reduced during the ramp which gradually lowers the threshold. If the same pulsed current is fed to the laser, the QCL starts emitting earlier and longer as the channel number increases. It is, however, for the present conditions below the threshold during at least half of the nominal current pulse width.

Taking into account a lower limit of $\sim 0.002 \mathrm{~cm}^{-1} / \mathrm{ns}$ for the chirp rate (e.g., [9]) an intrinsic and non-constant chirp of the pulses between 0.01 and $0.02 \mathrm{~cm}^{-1}$ is found which is even for these short pulses bigger than typical Doppler broadened absorption features. This is confirmed by Fig. 9 showing several individual QCL pulses during the laser sweep centred around feature II (i.e., channel 95 to 116): almost the entire $\mathrm{CH}_{4}$ line at $10 \mathrm{mbar}$ is present in the QCL pulse of channel 101. The example for channel 95 exhibits an amplified signal due to the rapid passage oscillations and channel 116 in Fig. 9 represents a nearly unaffected pulse at the high-frequency side of the sweep. However, all 20 pulses between channel 95 and 116 in Fig. 9 contain a non-negligible part of the $\mathrm{CH}_{4}$ line, which is often interpreted as an asymmetrical line broadening or line shape in the spectra recorded with the DAC. Extrapolating the trend in Fig. 9 strongly suggests that for QCLs having a higher chirp rate than the presently used device, absorption features spread over even more channels. In this case the asymmetry may be inverted and the shadow of the absorption line appears as a high-frequency tail if the laser intensity varies accordingly during the sweep (e.g., $[9,11])$.

\section{Summary}

A conventional short-pulse QCL spectrometer, where the laser sweep is achieved by an impressed bias current ramp as introduced in TDLAS, has been extended to perform a highly time-resolved analysis of individual laser pulses. While carefully chosen operating parameters for the laser might give correct results with the inter pulse method at elevated pressures ( $>50 \mathrm{mbar}$ ), this pictures changes entirely under low-pressure conditions. It transpires that the short-pulse mode exhibits all characteristics of the longpulse mode, particularly the rapid passage effect, but the tuning principle introduces further complications if applied to QCLs instead of TDLs. The oscillatory structure of the rapid passage effect is present in the deconvolved spectra, but not at first glance in conventionally acquired signals, which are characterised by broadened absorption features of reduced peak height.

These effects are more pronounced at higher laser intensities, i.e. clearly above the threshold, and for strong absorption features. Notably, all discussed examples were recorded not higher than $0.2 \mathrm{~V}$ above the threshold where the output power and hence the detector signal were just sufficient for achieving a reasonable SNR and thus the described obstacles can hardly be reduced. It is also clear that a generalised theoretical description is almost impossible to obtain since many parameters, such as the chirp rate or the temperature tuning coefficient, are strongly dependent on the single QCL device. The resulting total chirp in combination with the DC ramp parameters induce a shift of each spectral component over several detection channels. The total shift corresponds with what is normally considered as effective line width of pulsed QCLs $\left(\leq 0.05 \mathrm{~cm}^{-1}\right.$ or $1.5 \mathrm{GHz}$, FWHM). Additionally, the induced change of the QCL temperature by the bias 
current causes an increase of the pulse width and hence of the spectral chirp. The rapid passage effect is also more pronounced since the chirp rate is typically higher for the applied short pulses. The sum of all these individual contributions leads to asymmetrically broadened line shapes or even false absorption features which may be comparable to spectra obtained with multimode emitting lead salt lasers though the QCL is a single mode device. This behaviour, caused by the broad spectral coverage of a single chirped laser pulse, may therefore be considered as an undesirable and inevitable extended instrumental broadening.

However, it should be noted that the asymmetry is caused by a complex combination of (i) the gradient of the current ramp and intrinsic laser properties, i.e., (ii) the chirp rate of the QCL, and (iii) the intensity change during the sweep. This may lead to both low-frequency or high-frequency tails. The similarity between the conventionally acquired and artificially averaged spectra indicates that the entire experimental setup based on DACs which are conventionally used for TDLAS and LN detectors encompasses an unexpected timeaveraging of the signals or is limited in its bandwidth. This was also mentioned by Grouiez et al. [24] and will be scrutinised in a separate publication [31].

Acknowledgements The project was supported by the German Research Foundation (DFG) within the framework of the Collaborative Research Centre Transregio 24 "Fundamentals of Complex Plasmas". The authors thank Prof. P. Davies for useful discussions. The authors are indebted to $\mathrm{S}$. Glitsch for supplementary information and support with the QCL equipment. D. Gött, S. Saß and F. Weichbrodt provided valuable technical assistance.

Open Access This article is distributed under the terms of the Creative Commons Attribution Noncommercial License which permits any noncommercial use, distribution, and reproduction in any medium, provided the original author(s) and source are credited.

\section{References}

1. R.F. Kazarinov, R.A. Suris, Sov. Phys. Semicond. 5, 707 (1971)

2. J. Faist, F. Capasso, D.L. Sivco, C. Sirtori, A.L. Hutvhinson, A.Y. Cho, Science 264, 553 (1994)

3. F. Capasso, J. Faist, C. Sirtori, A.Y. Cho, Sol. State Commun. 102, 231 (1997)

4. K. Namjou, S. Cai, E.A. Whittaker, J. Faist, C. Gmachl, F. Capasso, D.L. Sivco, A.Y. Cho, Opt. Lett. 23, 219 (1998)

5. A.A. Kosterev, F.K. Tittel, C. Gmachl, F. Capasso, D.L. Sivco, J.N. Baillargeon, A.L. Hutchinson, A.Y. Cho, Appl. Opt. 39, 6866 (2000)

6. D.M. Sonnenfroh, W.T. Rawlins, M.G. Allen, C. Gmachl, F. Capasso, A.L. Hutchinson, D.L. Sivco, J.N. Baillargeon, A.Y. Cho, Appl. Opt. 40, 812 (2001)

7. D.D. Nelson, M.S. Zahniser, J.B. McManus, C.E. Kolb, J.L. Jimenez, Appl. Phys. B 67, 433 (1998)
8. M.S. Zahniser, D.D. Nelson, J.B. McManus, P.L. Kebabian, Philos. Trans. R. Soc. Lond. A 351, 371 (1995)

9. D.D. Nelson, J.H. Shorter, J.B. McManus, M.S. Zahniser, Appl. Phys. B 75, 343 (2002)

10. D.D. Nelson, J.B. McManus, S. Urbanski, S. Herndon, M.S. Zahniser, Spectrochim. Acta A 60, 3325 (2004)

11. Q. Shi, D.D. Nelson, J.B. McManus, M.S. Zahniser, M.E. Parrish, R.E. Baren, K.H. Shafer, C.N. Harward, Anal. Chem. 75, 5180 (2003)

12. J.B. McManus, D.D. Nelson, S.C. Herndon, J.H. Shorter, M.S. Zahniser, S. Blaser, L. Hvozdara, A. Muller, M. Giovannini, J. Faist, Appl. Phys. B 85, 235 (2006)

13. D. Weidmann, G. Wysocki, C. Oppenheimer, F.K. Tittel, Appl. Phys. B 80, 255 (2005)

14. J. Manne, W. Jäger, J. Tulip, Appl. Phys. B 94, 337 (2009)

15. A.A. Kosterev, R.F. Curl, F.K. Tittel, R. Köhler, C. Gmachl, F. Capasso, D.L. Sivco, A.Y. Cho, S. Wehe, M.G. Allen, Appl. Opt. 41, 1169 (2002)

16. G. Wysocki, M. McCurdy, S. So, D. Weidmann, C. Roller, R.F. Curl, F.K. Tittel, Appl. Opt. 43, 6040 (2004)

17. R.E. Baren, M.E. Parrish, K.H. Shafer, C.N. Harward, Q. Shi, D.D. Nelson, J.B. McManus, M.S. Zahniser, Spectrochim. Acta Part A 60, 3437 (2004)

18. G.D. Stancu, N. Lang, J. Röpcke, M. Reinicke, A. Steinbach, S. Wege, Chem. Vapor Depos. 13, 351 (2007)

19. F. Hempel, V. Artyushenko, F. Weichbrodt, J. Röpcke, J. Phys., Conf. Ser. 157, 012003 (2009)

20. N. Lang, J. Röpcke, H. Zimmermann, A. Steinbach, S. Wege, J. Phys., Conf. Ser. 157, 012007 (2009)

21. E. Normand, M. McCulloch, G. Duxbury, N. Langford, Opt. Lett. 28, 16 (2003)

22. T. Beyer, M. Braun, A. Lambrecht, J. Appl. Phys. 93, 3158 (2003)

23. M.T. McCulloch, E.L. Normand, N. Langford, G. Duxbury, D.A. Newnham, J. Opt. Soc. Am. B 20, 1761 (2003)

24. B. Grouiez, B. Parvitte, L. Joly, V. Zeninari, Opt. Lett. 34, 181 (2009)

25. G. Duxbury, N. Langford, M.T. McCulloch, S. Wright, Chem. Soc. Rev. 34, 921 (2005)

26. M.T. McCulloch, G. Duxbury, N. Langford, Mol. Phys. 104, 2767 (2006)

27. C. Gmachl, F. Capasso, R. Köhler, A. Tredicucci, A.L. Hutchinson, D.L. Sivco, J.N. Baillargeon, A.Y. Cho, IEEE Circuits Devices 16, 10 (2000)

28. G. Duxbury, N. Langford, M. McCulloch, S. Wright, Mol. Phys. 105, 741 (2007)

29. B. Grouiez, B. Parvitte, L. Joly, D. Courtois, V. Zeninari, Appl. Phys. B 90, 177 (2008)

30. J. Röpcke, L. Mechold, M. Käning, J. Anders, F.G. Wienhold, D. Nelson, M. Zahniser, Rev. Sci. Instrum. 71, 3706 (2000)

31. S. Welzel, J. Röpcke, (in preparation)

32. S. Welzel, Ph.D. Thesis, University of Greifswald (Logos, Berlin, 2009). ISBN 978-3-8325-2345-9

33. L.S. Rothman, D. Jacquemart, A. Barbe, D.C. Benner, M. Birk, L.R. Brown, M.R. Carleer, C. Chackerian, K. Chance, L.H. Coudert, V. Dana, V.M. Devi, J.M. Flaud, R.R. Gamache, A. Goldman, J.M. Hartmann, K.W. Jucks, A.G. Maki, J.Y. Mandin, S.T. Massie, J. Orphal, A. Perrin, C.P. Rinsland, M.A.H. Smith, J. Tennyson, R.N. Tolchenov, R.A. Toth, J. Vander Auwera, P. Varanasi, G. Wagner, J. Quant. Spectrosc. Radiat. Transf. 96, 139 (2005) 